Feb. 1996

IPM-96-138

hep-th/9604007

\title{
Logarithmic Operators in Conformal Field Theory and The $\mathcal{W}_{\infty}$-algebra
}

\author{
A. Shafiekhani ${ }^{a}$ ] and M.R. Rahimi Tabar ${ }^{a, b}$ \\ ${ }^{a}$ Institute for Studies in Theoretical Physics and Mathematics \\ P.O.Box: 19395-5531, Tehran, Iran \\ ${ }^{b}$ Dept. of Physics, Iran University of Science and Technology, \\ Narmak, Tehran, Iran
}

\begin{abstract}
It is shown explicitly that the correlation functions of Conformal Field Theories (CFT) with the logarithmic operators are invariant under the differential realization of Borel subalgebra of $\mathcal{W}_{\infty}$-algebra. This algebra is constructed by tensor-operator algebra of differential representation of ordinary $\operatorname{sl}(2, \mathbb{C})$. This method allows us to write differential equations which can be used to find general expression for three and four-point correlation functions possessing logarithmic operators. The operator product expansion (OPE) coefficients of general logarithmic CFT are given up to third level.
\end{abstract}

\footnotetext{
${ }^{1}$ e-mail address: ashafie@theory.ipm.ac.ir
} 


\section{Introduction}

It has been shown by Gurarie [1], that in the OPE of two given local fields which has at least two fields with the same conformal dimension, one can find some operators with a special property, known as logarithmic operators. As discussed in [1]], these operators together with the ordinary operators form the basis of the Jordan cell for the operator $L_{0}$. In some interesting physical theories, for example dynamics of polymers [2], WZW model on the $G L(1,1)$ super-group [3], percolation [4], edge excitation in fractional quantum Hall effect [5], one can find naturally such operators. Recently the role of such operators has been considered in the study of some physical problems, for instance: 2D-magnetohydrodynamic turbulence [6, 7, 8], 2D-turbulence [9, 10], $c_{p, 1}$ models [19, 11], gravitationally dressed CFT's [12, 13] and in some critical disordered models [15, 16]. They also play an important role in so called unifying $\mathcal{W}$ algebra [17] and in the description of normalizable zero modes for string backgrounds [14, 18.

The basic properties of logarithmic operators are, that they form part of the basis of the Jordan cell for $L_{0}$ whose in the correlators posses logarithmic singularity. It has been shown that in rational minimal models such a situation, i.e. two fields with the same conformal dimensions, does not occur [7]. The modular invariant partition functions for such theories with $c_{e f f}=1$ and the fusion rules of logarithmic conformal field theories (LCFT) are considered in [19, 20].

In this paper, we consider the symmetry algebra of the correlation functions with logarithmic factor. We take the modified operator $L_{0}$ according to ref. [1] and show that if certain conditions on the two point function of ordinary fileds with logarthmic partner is satisfied, such correlation functions remain invariant under $s l(2, \mathbb{C})$ algebra. Here, we introduce another method for calculating logarithmic operators. This method is obtained by finding differential equations from some combinations of ordinary $l_{n}$ 's differential realization. We show that this combination is related to the Borel subalgebra of the $\mathcal{W}_{\wedge}$-algebra, which is the wedge subalgebra of $\mathcal{W}_{\infty}$.

The structure of this paper is as follows: In section 2, we give a summary of LCFT and its relation to $\mathcal{W}_{\infty}$-algebra. In section 3 , we calculate the general behavior of three and 
four-point correlation functions of such theories. In section 4, we give the OPE coefficients up to third level of such CFT's.

\section{The Logarithmic Operators and $\mathcal{W}_{\infty}$-algebra}

According to [1], the OPE of two fields $A$ and $B$, which their fusion rule [21] contains two fields $\Phi$ and $\Psi$ of equal dimension, has a logarithmic term,

$$
A(z, \bar{z}) B(0,0)=|z|^{2\left(\Delta_{\Phi}-\Delta_{A}-\Delta_{B}\right)}\{\Psi(0,0)+\cdots+\log |z|[\Phi(0,0)+\cdots]\}
$$

where dots denote the descendants of fields $\Psi$ and $\Phi$. To see this, it is sufficient to look at the four-point function [21]:

$$
<A\left(z_{1}\right) B\left(z_{2}\right) A\left(z_{3}\right) B\left(z_{4}\right)>\sim \frac{1}{\left(z_{1}-z_{3}\right)^{2 \Delta_{A}}} \frac{1}{\left(z_{2}-z_{4}\right)^{2 \Delta_{B}}} \frac{1}{[x(1-x)]^{\Delta_{A}+\Delta_{B}-\Delta_{\Phi}}} F(x)
$$

where the cross ratio $x$ is given by:

$$
x=\frac{\left(z_{1}-z_{2}\right)\left(z_{3}-z_{4}\right)}{\left(z_{1}-z_{3}\right)\left(z_{2}-z_{4}\right)} .
$$

In degenerate models $F(x)$ satisfies a second order linear differential equation [21].

It should be noted that since logarithmic fields are not chiral, we can not separate holomorphic and anti-holomorphic parts. In this paper $\left(z_{i}-z_{j}\right)$ is set to $\left|z_{i}-z_{j}\right|^{2}$, for simplicity.

According to [7], the hypergeometric equation governing the correlator of the two fields in whose OPE, two other fields $\Psi$ and $\Phi$ with conformal dimension $\Delta_{\Psi}$ and $\Delta_{\Psi}+\epsilon$ appear, admits two solutions

$$
\begin{gathered}
{ }_{2} F_{1}(a, b, c, x) \\
x_{2}^{\epsilon} F_{1}(a+\epsilon, b+\epsilon, c+2 \epsilon, x)
\end{gathered}
$$

where $a, b$ and $c$ are sums of conformal dimension. Clearly in the limit of $\epsilon \rightarrow 0$ these two solutions coincide. However, it can be shown that [22] another independent solution exists which involves logarithms and can be generated by standard methods. Therefore, the following two independent solutions can be constructed according to $[1,10]$.

$$
\sum b_{n} x^{n}+\log x \sum a_{n} x^{n}
$$


Next, consistency of equation (11) and (2) requires that

$$
\begin{gathered}
<A\left(z_{1}\right) B\left(z_{2}\right) \Psi\left(z_{3}\right)>=<A\left(z_{1}\right) B\left(z_{2}\right) \Phi\left(z_{3}\right)>\left\{\log \frac{\left(z_{1}-z_{2}\right)}{\left(z_{1}-z_{3}\right)\left(z_{2}-z_{3}\right)}+\lambda\right\} \\
<\Psi(z) \Psi(0)>=-\frac{2}{z^{2 \Delta_{\Psi}}}\left[\log z+\lambda^{\prime}\right] \\
<\Psi(z) \Phi(0)>=\frac{1}{z^{2 \Delta_{\Phi}}}
\end{gathered}
$$

where $\lambda$ and $\lambda^{\prime}$ are constants. In this notation $\Psi$ is the logarithmic operator and $\Phi$ is the ordinary one. Let us now consider the action of $\operatorname{sl}(2, \mathbb{C})$ on the above correlators. In the absence of logarithmic operators (e.g., in the rational minimal models [21, 7]) the correlation function is invariant under the action of $\operatorname{sl}(2, \mathbb{C})$,

$$
\begin{gathered}
\sum_{i=1}^{N} \partial_{z_{i}}<\Phi_{1}\left(z_{1}\right) \cdots \Phi_{N}\left(z_{N}\right)>=0 \\
\sum_{i=1}^{N}\left(z_{i} \partial_{z_{i}}+\Delta_{i}\right)<\Phi_{1}\left(z_{1}\right) \cdots \Phi_{N}\left(z_{N}\right)>=0 \\
\sum_{i=1}^{N}\left(z_{i}^{2} \partial_{z_{i}}+2 z_{i} \Delta_{i}\right)<\Phi_{1}\left(z_{1}\right) \cdots \Phi_{N}\left(z_{N}\right)>=0
\end{gathered}
$$

Indeed according to [21, 23] the operators

$$
l_{-}=\partial_{z}, \quad l_{0}=z \partial_{z}+\Delta, \quad l_{+}=z^{2} \partial_{z}+2 z \Delta
$$

are the differential realization of $s l(2, \mathbb{C})$, with $\partial_{z}=\frac{\partial}{\partial z}$. When there are logarithmic operators in CFT theories, the ordinary correlation functions, for instance ordinary two-point function, should be replaced by eq. (8). According to [1], in the logarithmic conformal field theory, $l_{0}$ is given by the following modified representation:

$$
\left[L_{0}, A(z)\right]=\left(z \partial_{z}+\Delta_{A}+\mathcal{D}\right) A(z)
$$

where the operator $\mathcal{D}$ is such that $\mathcal{D} \Phi(z) \equiv 0$ and $\mathcal{D} \Psi(z) \equiv \Phi$. This can be constructed by taking the OPE of $T(z)$ with $\Phi(0)$ and with $\Psi(0)$. That is to say

$$
\begin{gathered}
T(z) \Psi(0)=\frac{\Delta}{z^{2}} \Psi(0)+\frac{\partial_{z} \Psi(0)}{z}+\frac{\Phi(0)}{z^{2}}+\cdots, \\
T(z) \Phi(0)=\frac{\Delta}{z^{2}} \Phi(0)+\frac{\partial_{z} \Phi(0)}{z}+\cdots .
\end{gathered}
$$


If we expand $T(z)$ by Laurant series we can write [1],

$$
\begin{gathered}
{\left[l_{n}, \Psi(z)\right]=\left(z^{n+1} \partial_{z}+\Delta(n+1) z^{n}\right) \Psi(z)+(n+1) z^{n} \Phi(z)} \\
{\left[l_{n}, \Phi(z)\right]=\left(z^{n+1} \partial_{z}+\Delta(n+1) z^{n}\right) \Phi(z)}
\end{gathered}
$$

which means that we can redefine $l_{n}$ to $L_{n}$ such that:

$$
\left[L_{n}, A(z)\right]=\left(z^{n+1} \partial_{z}+\Delta_{A}(n+1) z^{n}+(n+1) z^{n} \mathcal{D}\right) A(z)
$$

with the property $\mathcal{D} \Psi \equiv \Phi$ and $\mathcal{D} \Phi \equiv 0$. For example, one can show that eq.(8) is invariant under modified $L_{0}$ ( i.e. non-diagonal $L_{0}$ ).

$$
\begin{gathered}
L_{0}<\Psi(z) \Psi(0)>=<\left[L_{0}, \Psi(z) \Psi(0)\right]> \\
=<\left[\left(z \partial_{z}+\Delta_{\Psi}\right) \Psi(z)+\Phi(z)\right] \Psi(0)>+<\Psi(z) \Delta_{\Psi} \Psi(0)>+<\Psi(z) \Phi(0)> \\
=\left(z \partial_{z}+2 \Delta_{\Psi}\right)<\Psi(z) \Psi(0)>+<\Psi(z) \Phi(0)>+<\Phi(z) \Psi(0)>.
\end{gathered}
$$

By using eqs.(8) and (9) we find that:

$$
L_{0}<\Psi(z) \Psi(0)>=0
$$

Conversely, the eq.(19) allows us to write Ward identities (for $n=-1,0,1$ ) for the calculation of the correlation functions. For instance, for two-point functions we have:

$$
\begin{gathered}
L_{-1}<\Psi(z) \Psi(0)>=L_{-1}<\Psi(z) \Phi(0)>=L_{-1}<\Phi(z) \Phi(0)>=0 \\
L_{0}<\Psi(z) \Psi(0)>=L_{0}<\Psi(z) \Phi(0)>=L_{0}<\Phi(z) \Phi(0)>=0 \\
L_{+}<\Psi(z) \Psi(0)>=L_{+}<\Psi(z) \Phi(0)>=L_{+}<\Phi(z) \Phi(0)>=0 .
\end{gathered}
$$

Using eq.(23) we find,

$$
\begin{gathered}
\left(z \partial_{z}+2 \Delta_{\Psi}\right)<\Psi(z) \Psi(0)>+2<\Psi(z) \Phi(0)>=0 \\
\left(z \partial_{z}+2 \Delta_{\Psi}\right)<\Psi(z) \Phi(0)>+<\Psi(z) \Phi(0)>=0 \\
\left(z \partial_{z}+2 \Delta_{\Psi}\right)<\Phi(z) \Phi(0)>=0
\end{gathered}
$$

which can be solved yeilding:

$$
<\Phi(z) \Phi(0)>=-\frac{a}{z^{2 \Delta_{\Psi}}}
$$




$$
\begin{gathered}
<\Psi(z) \Phi(0)>=\frac{1}{z^{2 \Delta_{\Psi}}}\left[2 a \log z+a^{\prime}\right] \\
<\Psi(z) \Psi(0)>=\frac{1}{z^{2 \Delta_{\Psi}}}\left[2 a \log ^{2} z-2 a^{\prime} \log z+a^{\prime \prime}\right]
\end{gathered}
$$

where so far $a, a^{\prime}$ and $a^{\prime \prime}$ are arbitry parameters. One can show that eq.(24) leads to the following relation:

$$
<\Phi(z) \Phi(0)>=0
$$

or $a=0$ which has already been pointed out in [15]. This means that if we insist on having conformal invariance, the two-point function of ordinary fields which have logarithmic partner must be zero.

For $a \neq 0$ we don't have the conformal invariance, however the correlation functions are invariant under $L_{+}$and $L_{-}$, which is the subalgebra of $\operatorname{sl}(2, \mathbb{C})$. The condition $(31)$ seems to be very restrictive on two-point correlation functions. For example, in $c=-2$ theory, which belongs to LCFT's of $c_{p, 1}$ series (with $\mathrm{p}=2$ ) [20], the requirment of the conformal invariance forced sets the two-point functions of fields with logarithmic partner to zero. In other words, the ordinary fields with logarithmic partners do not propagate.

Here, we introduce another method for finding certain differential equations which can be used to obtain the correlation functions by means of ordinary $l_{n}$ 's. This approach is based on the simple observation that in applying the differential operator $\left(z \partial_{z}+2 \Delta_{\Psi}\right)$ (the differential representation of $l_{0}$ ) on eq.(8), we obtain:

$$
l_{0}<\Psi(z) \Psi(0)>=<\left[l_{0}, \Psi(z) \Psi(0)\right]>\equiv\left(z \partial_{z}+2 \Delta_{\Psi}\right)<\Psi(z) \Psi(0)>=-2 z^{-2 \Delta_{\Psi}} .
$$

which behaves as eq.(9). Similary the action of ordinary $l_{ \pm}$are as follows:

$$
\begin{gathered}
<\left[l_{-1}, \Psi(z) \Psi(0)\right]>\equiv\left(\partial_{z}\right)<\Psi(z) \Psi(0)>=0 \\
<\left[l_{+}, \Psi(z) \Psi(0)\right]>\equiv\left(z^{2} \partial_{z}+2 z \Delta_{\Psi}\right)<\Psi(z) \Psi(0)>=-2 z^{-2 \Delta_{\Psi}+1} .
\end{gathered}
$$

Now we consider the action of $l_{0}$ and $l_{+}$on the correlation of $\langle\Psi(z) \Psi(0)>$. This can be written in the following form:

$$
<\left[l_{0},\left[l_{+}, \Psi(z) \Psi(0)\right]\right]>=\left(z^{2} \partial_{z}+2 z \Delta_{\Psi}\right)\left(z \partial_{z}+2 \Delta_{\Psi}\right)<\Psi(z) \Psi(0)>=
$$




$$
\left(z^{2} \partial_{z}+2 z \Delta_{\Psi}\right)\left(z \partial_{z}+2 \Delta_{\Psi}\right)\left(-\frac{2}{z^{2 \Delta_{\Psi}}}\left[\log z+\lambda^{\prime}\right]\right)=0
$$

Indeed, this is the action of differential representation of $l_{0}$ and $l_{+}$on the logarithmic twopoint function.

At first glance, it seems that the logarithmic correlation function, $<\Psi\left(z_{1}\right) \Psi\left(z_{2}\right)>$ is invariant under the set of

$$
\left\{l_{-}, l_{0}^{2}, l_{-} l_{0}, l_{+} l_{0}, l_{0} l_{-} l_{+}, l_{-}^{2} l_{+}, l_{+} l_{-} l_{+}\right\}
$$

where the last three of them are observed using the fact that $l_{-} l_{+}$times such correlation functions behave as ordinary correlation functions in CFT. Using the commutation relation of $l_{0}, l_{+}$and $l_{-}$

$$
\left[l_{0}, l_{ \pm}\right]= \pm l_{ \pm} \quad\left[l_{+}, l_{-}\right]=-2 l_{0}
$$

the last three members of Eq. (25) can be written in terms of the first four which we call $(A, B, C, D)$ respectively.

Simple calculations show that, the following algebraic relations hold:

$$
[A, B]=-2 C-A, \quad[B, C]=2 A B+C, \quad[C, D]=-2 l_{0}^{3}-2 l_{0} \cdots
$$

Explicit calculation shows that this algebra is not closed. In each step, we need to add new operators to the algebra.

On the other hand, the action of $l_{0}$ on logarithmic correlation functions behave as expected. However the two-point function is the exception. In the case of three-point and four-point correlation functions, the action of differential representation of $l_{0}$ on correlation functions which have logarithmic term, behave like ordinary three-point and four-point functions of CFT. We will come to this point in the next section.

In the rest of this section we determine the connection between the above algebra and the infinite dimensional $\mathcal{W}_{\infty}$-algebra. The algebraic commutation relation of $V^{i}$ 's, generators of spin- $(i+2) \mathcal{W}_{\infty}$-algebra, are as follow [24]:

$$
\left[V_{m}^{i}, V_{n}^{j}\right]=\sum_{l \geq 0} g_{2 l}^{i j}(m . n) V_{m+n}^{i+j-2 l}+c_{i}(m) \delta^{i, j} \delta_{m+n, 0}
$$

where $g_{2 l}^{i j}(m, n)$ are structure constants of the algebra and $c_{i}(m)$ are central terms. $V_{m}^{i}$ denotes the $m$ th Laurent mode of spin- $(i+2)$ current $V^{i}(z)$. In our case, this current can be 
constructed from the logarithmic operator $\Psi$ [14]. For $\mathcal{W}_{\infty}$-algebra with spins $2 \leq s<\infty$, the indices $i$ and $j$ range from 0 to $\infty$. The structure constants and central terms can be determined by demanding that eq. (38) be consistent with the Jacobi identities [24, 25],

$$
c_{i}(m)=m\left(m^{2}-1\right)\left(m^{2}-4\right) \cdots\left(m^{2}-(i+2)^{2}\right) c_{i}
$$

where $c_{i}$ 's are central charges, and the structure constants take the form

$$
g_{l}^{i j}(m, n)=\frac{1}{2(l+1) !} \Phi_{l}^{i j} N_{l}^{i j}(m, n)
$$

where $N_{l}^{i j}(m, n)$ are given by

$$
N_{l}^{i j}(m, n)=\sum_{k=0}^{l+1}(-1)^{k}\left(\begin{array}{l}
l+1 \\
k
\end{array}\right)[i+1+m]_{l+1-k}[i+1-m]_{k}[j+1+n]_{k}[j+1-n]_{l=1-k}
$$

and $[a]_{n}=a(a-1) \cdots(a-n+1)=\frac{a !}{(a-n) !}$. The functions $\Phi_{l}^{i j}$ are given by

$$
\Phi_{l}^{i j}={ }_{4} F_{3}\left[\begin{array}{cc}
-\frac{1}{2}, \frac{3}{2},-\frac{l}{2}-\frac{1}{2},-\frac{l}{2} & ; 1 \\
-i-\frac{1}{2},-j-\frac{1}{2}, i+j-l+\frac{5}{2} &
\end{array}\right]
$$

where ${ }_{4} F_{3}(1)$ is a generalized hypergeometric function [25]. Now consider a "wedge" of generators $V_{m}^{i}$ for which $|m| \leq(i+1)$. It can be easily shown that the commutator of any two generators within a wedge only involves generators within the same wedge. The resulting wedge subalgebra of $\mathcal{W}_{\infty}$ which is known as $\mathcal{W}_{\wedge}$, has the feature that it is anomaly free, the central terms vanish for all commutators. The algebra $\mathcal{W}_{\wedge}$ can be realized by generalization of $s l(2)$, subalgebra of the Virasoro algebra, generated by $l_{+}, l_{-}$and $l_{0}$. Let us consider the tensor-operator algebra of $s l(2, \mathbb{C})$. The tensor operator algebra may be constructed in the following way. The $\operatorname{sl}(2, \mathbb{C})$ generators satisfy the commutation relations of eqs. (36). The Casimir operator may be written as

$$
Q=l_{0}^{2}-\frac{1}{2}\left(l_{+} l_{-}+l_{-} l_{+}\right) .
$$

The three generators, $l_{-}, l_{+}$and $l_{0}$ transform as the 3 representations of $\operatorname{sl}(2, \mathbb{C})$. Highertensor operators, $T_{m}^{l}$ with $(-l \leq m \leq l)$, transforming in general as the $(2 l+1)$-dimensional representation of $s l(2, \mathbb{C})$, are constructed from appropriate polynomials of degree $l$ in the 
l's. According to 25], we start with the highest-weight state $T_{l}^{l}=\left(l_{+}\right)^{l}$, and then construct the lower-weight states in the usual way by $(l-m)$ successive application of $L_{-}$,

$$
T_{m}^{l}=\frac{1}{(-2 l)_{l-m}}\left[l_{-},\left[l_{-}, \cdots\left[l_{-},\left(l_{+}\right)^{l}\right] \cdots\right]\right]
$$

where $(a)_{n}=a(a+1) \cdots(a+n-1)=\frac{(a+n-1) !}{(a-1) !}$. Subtilties arise when we determine the exact relation of the above construction with the $\mathcal{W}_{\infty}$-algebra. Acting upon a Hilbert space in which the quadratic Casimir takes on a definite value $Q=\mu$, the operators $T_{m}^{l}$ close into an infinite-dimensional algebra which is parametrized by $\mu$, and known as $\mathcal{T}(\mu)$. Therefore, we would expect that the above construction for $\mathcal{W}_{\wedge}$-algebra should coincide with $\mathcal{T}(\mu)$ for some specific value of $\mu$. It has been shown in ref. [24], that the wedge subalgebra $\mathcal{W}_{\wedge}$ contained in $\mathcal{W}_{\infty}$-algebra is the $\operatorname{sl}(2, \mathbb{C})$ operator algebra $\mathcal{T}(0)$, specified by the value $\mu=0$ for the quadratic Casimir. However for any value of the parameter $\mu$, we can allow the representation to be infinite dimensional [24]. It can be easily checked that in our case the logarithmic correlation functions are not invariant under all of $T_{m}^{l}$ 's, but they are invariant under $T_{m}^{l}$ with $-l \leq m \leq 0$. Therefore the important result is that the logarithmic correlation functions are invariant under the Borel subalgebra of $\mathcal{W}_{\wedge}$-algebra.

\section{Correlation Functions with Logarithmic Behavior}

In this section, we calculate the correlation functions that have a logarithmic term. A straightforward way for calculating the correlation functions is to use Ward identities [26]. For simplicity, first we consider the correlation functions of conformal fields where only two of them have two fields of equal dimension in their OPE. This can be done by using the corresponding symmetry algebra, which was found in the last section. It can be shown that in this case all correlation functions behave as follows:

$$
l_{0}<A\left(z_{1}\right) B\left(z_{2}\right) \mathcal{O}\left(z_{3}\right) \cdots \mathcal{O}\left(z_{n}\right)>=k_{n}<\mathcal{O}\left(z_{1}\right) \cdots \mathcal{O}\left(z_{n}\right)>
$$

where the OPE of $A\left(z_{n-1}\right) B\left(z_{n}\right)$ is given by eq. (1) and $\mathcal{O}\left(z_{i}\right)$ are ordinary operators. For the calculation of the two point function of such a conformal field theory, we have

$$
l_{0}<\Psi\left(z_{1}\right) \Psi\left(z_{2}\right)>=k_{2}<\mathcal{O}\left(z_{1}\right) \mathcal{O}\left(z_{2}\right)>=k_{2} \frac{1}{z_{12}^{2 \Delta_{\Psi}}}
$$


and the following Ward identities:

$$
l_{0} l_{0}<\Psi\left(z_{1}\right) \Psi\left(z_{2}\right)>=0, \quad l_{-}<\Psi\left(z_{1}\right) \Psi\left(z_{2}\right)>=0, \quad l_{+} l_{0}<\Psi\left(z_{1}\right) \Psi\left(z_{2}\right)>=0,
$$

and so on, where $z_{i j}=z_{i}-z_{j}$. Now we substitute the differential realization of $l_{0}$ and $l_{ \pm}$ from (13) into the above equations by means of the "co-product",

$$
\begin{gathered}
{\left[z_{1} \partial_{z_{1}}+z_{2} \partial_{z_{2}}+2 \Delta_{\Psi}\right]<\Psi\left(z_{1}\right) \Psi\left(z_{2}\right)>=k_{2}<\mathcal{O}\left(z_{1}\right) \mathcal{O}\left(z_{2}\right)>} \\
{\left[\left(z_{1} \partial_{z_{1}}+z_{2} \partial_{z_{2}}+2 \Delta_{\Psi}\right)\left(z_{1} \partial_{z-1}+z_{2} \partial_{z_{2}}+2 \Delta_{\Psi}\right)\right]<\Psi\left(z_{1}\right) \Psi\left(z_{2}\right)>=0} \\
{\left[\partial_{z_{1}}+\partial_{z_{2}}\right]<\Psi\left(z_{1}\right) \Psi\left(z_{2}\right)>=0} \\
{\left[z_{1}^{2} \partial_{z_{1}}+z_{2}^{2} \partial_{z_{2}}+2\left(z_{1}+z_{2}\right) \Delta_{\Psi}\right]<\Psi\left(z_{1}\right) \Psi\left(z_{2}\right)>=0}
\end{gathered}
$$

and so on. Solving the above equations for $<\Psi\left(z_{1}\right) \Psi\left(z_{2}\right)>$, we have

$$
<\Psi\left(z_{1}\right) \Psi\left(z_{2}\right)>=k_{2}<\mathcal{O}\left(z_{1}\right) \mathcal{O}\left(z_{2}\right)>\left[\log z_{12}+\lambda^{\prime}\right]
$$

where $k_{2}$ is -2 and $\lambda^{\prime}$ is a constant.

A similar methods can be used for solving the three point correlation function. Instead of $(46)$ we have

$$
l_{0}<A\left(z_{1}\right) B\left(z_{2}\right) \Psi\left(z_{3}\right)>=k_{3}<A\left(z_{1}\right) B\left(z_{2}\right) \Phi\left(z_{3}\right)>=\frac{k_{3}}{z_{12}^{\Delta_{A}+\Delta_{B}-\Delta_{\Psi}} z_{13}^{\Delta_{A}-\Delta_{B}+\Delta_{\Psi}} z_{23}^{-\Delta_{A}+\Delta_{B}+\Delta_{\Psi}}}
$$

and similar Ward identities for the three-point function. Solving these equations gives:

$$
<A\left(z_{1}\right) B\left(z_{2}\right) \Psi\left(z_{3}\right)>=k_{3}<A\left(z_{1}\right) B\left(z_{2}\right) \Phi\left(z_{3}\right)>\left(a \log z_{12}+b \log z_{13}+c \log z_{23}+\lambda\right)
$$

where $k_{3}, a, b$ and $c$ must satisfy the following relation:

$$
k_{3}+a+b+c=0 .
$$

For the four-point correlation function, $<A\left(z_{1}\right) B\left(z_{2}\right) \mathcal{O}\left(z_{3}\right) \mathcal{O}\left(z_{4}\right)>$, we have

$$
\begin{aligned}
& L_{0}<A\left(z_{1}\right) B\left(z_{2}\right) \mathcal{O}_{1}\left(z_{3}\right) \mathcal{O}_{2}\left(z_{4}\right)>=k_{4}<\mathcal{O}\left(z_{1}\right) \mathcal{O}\left(z_{2}\right) \mathcal{O}\left(z_{3}\right) \mathcal{O}\left(z_{4}\right)>
\end{aligned}
$$

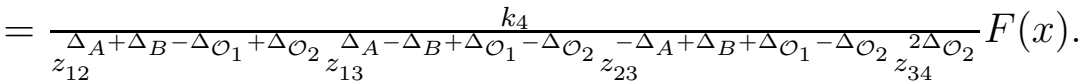

Solving the relevant equations by a similar method, we find

$$
\begin{gathered}
<A\left(z_{1}\right) B\left(z_{2}\right) \mathcal{O}\left(z_{3}\right) \mathcal{O}\left(z_{4}\right)>=k_{4}<\mathcal{O}\left(z_{1}\right) \mathcal{O}\left(z_{2}\right) \mathcal{O}\left(z_{3}\right) \mathcal{O}\left(z_{4}\right)> \\
\times\left[a \log z_{12}+b \log z_{13}+c \log z_{14}+d \log z_{23}+e \log z_{24}+f \log z_{34}+\lambda^{\prime \prime}\right]
\end{gathered}
$$


where $\lambda "$ is a constant and $k_{4}, a, b, c, d, e$ and $f$ must satisfy:

$$
k_{4}+a+b+c+d+e+f=0 .
$$

It can be seen that eq. (54), is valid for four-point function of $<A\left(z_{1}\right) B\left(z_{2}\right) \mathcal{O}\left(z_{3}\right) \Psi\left(z_{4}\right)>$ type. However correlation function of $<\Psi\left(z_{1}\right) \Psi\left(z_{2}\right) \Psi\left(z_{3}\right)>$ type cannot be calculated by this method [27].

\section{OPE Coefficients}

\subsection{OPE of Ordinary Conformal Fields}

The most general expression for the operator product expansion of ordinary conformal fields is [21, []:

$$
\Phi_{n}(z, \bar{z}) \Phi_{m}(0,0)=\sum_{p} \sum_{k} z^{\Delta_{p}-\Delta_{n}-\Delta_{m}+\sum k_{i}} \bar{z}^{\bar{\Delta}_{p}-\bar{\Delta}_{n}-\bar{\Delta}_{m}+\sum \bar{k}_{i}} C_{n m}^{p,\{k\},\{\bar{k}\}} \Phi_{p}^{\{k\},\{\bar{k}\}}(0,0)
$$

where the coefficients are

$$
C_{n m}^{p,\{k\},\{\bar{k}\}}=C_{n m}^{p} \beta_{n m}^{p,\{k\}} \bar{\beta}_{n m}^{p,\{\bar{k}\}}, \quad\{k\}=\left\{k_{1}, k_{2}, \cdots, k_{n}\right\}
$$

This OPE is the first regular hypergeometric function $F(x)$. Note that we have $\Phi_{i}(0)|0>=|$ $\Delta_{i}>$. Acting on $\mid 0>$, by eq. (56) we obtain:

$$
\begin{gathered}
\Phi_{n}(z)\left|\Delta_{m}>=\sum_{p} C_{n m}^{p} z^{\Delta_{p}-\Delta_{n}-\Delta_{m}} \phi_{p}(z)\right| \Delta_{p}> \\
\phi_{p}(z)=\sum_{k} z^{\sum k_{i}} \beta_{n m}^{p,\{k\}} L_{-k_{1}} \cdots L_{-k_{n}} \\
\left|z, \Delta_{p}>=\phi_{p}(z)\right| \Delta_{p}>.
\end{gathered}
$$

Expanding $\mid z, \Delta_{p}>$ in terms of the complete basis $\mid N, \Delta_{p}>$, i.e.,

$$
\left|z, \Delta_{p}>=\sum_{N} z^{N}\right| N, \Delta_{p}>
$$

and applying $L_{j}$ on eq. (58), we obtain:

$$
L_{j}\left|N+j, \Delta_{p}>=\left(\Delta_{p}-\Delta_{m}+j \Delta_{n}+N\right)\right| N, \Delta_{p}>
$$


We also had that

$$
L_{0}\left|N, \Delta_{p}>=\left(\Delta_{p}+N\right)\right| N, \Delta_{p}>.
$$

By solving the recursion relations we can find $\beta_{n m}^{p,\{k\}}$.

For the first level we have

$$
L_{1}\left|1, \Delta_{p}>=\left(\Delta_{p}-\Delta_{m}-\Delta_{n}\right)\right| \Delta_{p}>
$$

which results in

$$
\left|1, \Delta_{p}>=\alpha_{1} L_{-1}\right| \Delta_{p}>; \quad \alpha_{1}=\frac{\Delta_{p}-\Delta_{m}+\Delta_{n}}{2 \Delta_{p}} .
$$

For the second level by means of eqs. (60) and (64) we have

$$
\begin{gathered}
L_{1}\left|2, \Delta_{p}>=\hat{A} \alpha_{1} L_{-1}\right| \Delta_{p}> \\
L_{2}\left|2, \Delta_{p}>=\hat{B}\right| \Delta_{p}>
\end{gathered}
$$

which results in:

$$
\left|2, \Delta_{p}>=\left(\alpha_{2} L_{-1}^{2}+\alpha_{3} L_{-2}\right)\right| \Delta_{p}>
$$

with $\alpha_{2}$ and $\alpha_{3}$ satisfying the following system of equations:

$$
{ }_{2} \mathcal{N}_{i j} \alpha_{j}={ }_{2} \mathcal{A}_{i}, \quad i, j=2,3
$$

where

$$
{ }_{2} \mathcal{N}=\left[\begin{array}{cc}
4 \Delta_{p}+2 & 3 \\
6 \Delta_{p} & 4 \Delta_{p}+\frac{c}{2}
\end{array}\right], \quad{ }_{2} \mathcal{A}=\left[\begin{array}{c}
\hat{A} \alpha_{1} \\
\hat{B}
\end{array}\right] .
$$

and index "2" refers to the level. This system can now be solved to give:

$$
\left[\begin{array}{l}
\alpha_{2} \\
\alpha_{3}
\end{array}\right]=\frac{1}{\Delta_{1}}\left[\begin{array}{c}
\hat{C}-3 \hat{B} \\
-6 \Delta_{p} \hat{A} \alpha_{1}+\hat{D} \hat{B}
\end{array}\right]
$$

where

$$
\begin{aligned}
& \hat{A}=\Delta_{p}-\Delta_{m}+\Delta_{n}+1 \\
& \hat{B}=\Delta_{p}-\Delta_{m}+2 \Delta_{n} \\
& \hat{C}=4 \Delta_{p}+\frac{c}{2} \\
& \hat{D}=4 \Delta_{p}+2
\end{aligned}
$$




$$
\Delta_{1}=c\left(2 \Delta_{p}+1\right)+2 \Delta_{p}\left(8 \Delta_{p}-5\right) .
$$

A similar method will work for higher levels. For the level N, we have in place of eq. (66), an expansion corresponding to the partition of $\mathrm{N}$. We then find a system of equations by successively applying $L_{j}$, and finally the coefficients are derived. For the third level, using Eq. (61), we have:

$$
\begin{aligned}
& L_{1}\left|3, \Delta_{p}>=(\hat{A}+1)\left(\alpha_{2} L_{-2}+\alpha_{3} L_{-1}^{2}\right)\right| \Delta_{p}> \\
& L_{2}\left|3, \Delta_{p}>=(\hat{B}+1) \alpha_{1} L_{-1}\right| \Delta_{p}> \\
& L_{3}\left|3, \Delta_{p}>=\left(\hat{B}+\Delta_{n}\right)\right| \Delta_{p}>
\end{aligned}
$$

and $\mid 3, \Delta_{p}>$ is given by

$$
\left|3, \Delta_{p}>=\left(\alpha_{4} L_{-1}^{3}+\alpha_{5} L_{-1} L_{-2}+\alpha_{6} L_{-3}\right)\right| \Delta_{p}>
$$

where $\alpha_{4}, \alpha_{5}$ and $\alpha_{6}$ satisfy the following system of equations:

$$
{ }_{3} \mathcal{N}_{i j} \alpha_{j}={ }_{3} \mathcal{A}_{i}, \quad i, j=4,5,6
$$

with

$$
{ }_{3} \mathcal{N}=\left[\begin{array}{ccc}
18 \Delta_{p}+6 & 4 \Delta_{p}+\frac{c}{2}+9 & 5 \\
24 \Delta_{p} & 16 \Delta_{p}+2 c & 6 \Delta_{p}+2 c \\
6 \Delta_{p}+6 & 2 \Delta_{p}+7 & 4
\end{array}\right] \quad{ }_{3} \mathcal{A}=\left[\begin{array}{c}
(\hat{B}+1) \alpha_{1} \\
\hat{B}+\Delta_{n} \\
(\hat{A}+1)\left(\alpha_{2}+\alpha_{3}\right)
\end{array}\right]
$$

Solving of the above system we obtain:

$$
\begin{gathered}
\alpha_{4}=\frac{-1}{6 \Delta_{2}}\left[2 \alpha_{1}(\hat{B}+1)\left(6 \Delta_{p}^{2}+6 c \Delta_{p}-11 \Delta_{p}+3 c\right)+\left(\hat{B}+\Delta_{n}\right)\left(6 \Delta_{p}+2 c+1\right)\right. \\
\left.-(\hat{A}+1)\left(\alpha_{2}+\alpha_{3}\right)\left(24 \Delta_{p}^{2}+11 c \Delta_{p}-26 \Delta_{p}+c^{2}+8 c\right)\right] \\
\alpha_{5}=\frac{1}{\Delta_{2}}\left[2 \alpha_{1}(\hat{B}+1)\left(3 \Delta_{p}^{2}+c \Delta_{p}-5 \Delta_{p}+c\right)+\left(\hat{B}+\Delta_{n}\right)\left(7 \Delta_{p}-1\right)\right. \\
\left.-2(\hat{A}+1)\left(\alpha_{2}+\alpha_{3}\right)\left(9 \Delta_{p}^{2}+3 c \Delta_{p}-7 \Delta_{p}+c\right)\right] \\
\alpha_{6}=\frac{1}{2 \Delta_{2}}\left[-4(\hat{B}+1) \alpha_{1}\left(4 \Delta_{p}^{2}+c \Delta_{p}-\Delta_{p}+c\right)+\left(\hat{B}+\Delta_{n}\right)\left(-4 \Delta_{p}^{2}+c \Delta_{p}\right.\right. \\
\left.\left.-20 \Delta_{p}+c+4\right)+4(\hat{A}+1)\left(\alpha_{2}+\alpha_{3}\right)\left(16 \Delta_{p}^{2}+2 c \Delta_{p}-10 \Delta_{p}+c\right)\right]
\end{gathered}
$$

where

$$
\Delta_{2}=c^{2}\left(\Delta_{p}+1\right)-c\left(\Delta_{p}^{2}+11 \Delta_{p}-2\right)-4 \Delta_{p}\left(3 \Delta_{p}^{2}-7 \Delta_{p}+2\right) .
$$




\subsection{OPE of Logarithmic Conformal Fields}

The second most general solution of $F(x)$, which implies new OPE for two conformal fields which have two fields $\phi$ and $\psi$, of equal dimension in their fusion rule, eq. (1), is:

$$
\begin{gathered}
\Phi_{n}(z, \bar{z}) \Phi_{m}(0,0)=\sum_{p} \sum_{k} z^{\Delta_{p}-\Delta_{n}-\Delta_{m}+\sum k_{i}} \bar{z}^{\bar{\Delta}_{p}-\bar{\Delta}_{n}-\bar{\Delta}_{m}+\sum \bar{k}_{i}} \\
\quad \times\left[C_{n m}^{p,\{k\},\{\bar{k}\}} \log |z|^{2} \phi_{p}^{\{k\},\{\bar{k}\}}(0,0)+C_{n m}^{\prime p,\{k\},\{\bar{k}\}} \psi_{p}^{\{k\},\{\bar{k}\}}(0,0)\right]
\end{gathered}
$$

where $\phi_{p}^{\{k\},\{\bar{k}\}}$ is an ordinary conformal fields, which has been discussed in the last subsection and $\psi_{p}^{\{k\},\{\bar{k}\}}$ denote the new pseudo-operator with unusual properties.

The coefficients $C_{n m}^{p,\{k\},\{\bar{k}\}}$ are the same as eq.(57) and

$$
C_{n m}^{\prime p,\{k\},\{\bar{k}\}}=C_{n m}^{\prime p} \beta_{n m}^{p,\{k\}} \bar{\beta}_{n m}^{p,\{\bar{k}\}} \quad\{k\}=\left\{k_{1}, k_{2}, \cdots, k_{n}\right\} .
$$

Similar to the expressions for $\phi_{p}^{\{k\},\{\bar{k}\}}$, we have the following for $\psi_{p}^{\{k\},\{\bar{k}\}}$ :

$$
\psi_{i}(0)|0>=| \Delta_{p}^{\prime}>,\left|z, \Delta_{p}^{\prime}>=\psi_{p}(z)\right| \Delta_{p}^{\prime}>
$$

and instead of eq. (58), we have

$$
\phi_{n}(z)\left|\Delta_{m}>=\sum_{p} z^{\Delta_{p}-\Delta_{n}-\Delta_{m}}\left[\log z C_{n m}^{p} \phi_{p}(z)+C_{n m}^{\prime p} \psi_{p}(z)\right]\right| \Delta_{p}>
$$

where

$$
\left|z, \Delta_{p}^{\prime}>=\psi_{p}(z)\right| \Delta_{p}>
$$

and its expansion in terms of $z^{N}$ is

$$
\left|z, \Delta_{p}^{\prime}>=\sum_{N} z^{N}\right| N, \Delta_{p}^{\prime}>
$$

Collecting the above expressions together, we obtain the following relations [1, 6, 21]

$$
\begin{gathered}
L_{j}\left|N+j, \Delta_{p}>=\left(\Delta_{p}-\Delta_{m}+j \Delta_{n}+N\right)\right| N, \Delta_{p}> \\
L_{j}\left|N+j, \Delta_{p}^{\prime}>=\right| N, \Delta_{p}>+\left(\Delta_{p}-\Delta_{m}+j \Delta_{n}+N\right) \mid N, \Delta_{p}^{\prime}> \\
L_{0}\left|N, \Delta_{p}>=\left(N+\Delta_{p}\right)\right| N, \Delta_{p}>. \\
L_{0}\left|N, \Delta_{p}^{\prime}>=\right| N, \Delta_{p}>+\left(\Delta_{p}+N\right) \mid N, \Delta_{p}^{\prime}>.
\end{gathered}
$$


Now by using (82-85), we can calculate the OPE coefficients of two conformal operators, which have at least two operators with equal dimension in their OPE.

In the same way as in the ordinary case, for the first level we have

$$
\begin{gathered}
\left|1, \Delta_{p}^{\prime}>=\alpha_{1}^{\prime} L_{-1}\right| \Delta_{p}>+\beta_{1} L_{-1} \mid \Delta_{p}^{\prime}> \\
L_{1}\left|1, \Delta_{p}^{\prime}>=\left(2 \Delta_{p} \alpha_{1}^{\prime}+2 \beta_{1}\right)\right| \Delta_{p}>+2 \Delta_{p} \beta_{1} \mid \Delta_{p}^{\prime}> \\
=\left|\Delta_{p}>+\left(\Delta_{p}-\Delta_{m}+\Delta_{n}\right)\right| \Delta_{p}^{\prime}>
\end{gathered}
$$

with $\alpha^{\prime}{ }_{1}$ and $\beta_{1}$ satisfying the following system of equations;

$$
{ }_{1} \mathcal{M}_{i j} \gamma_{j}={ }_{1} \mathcal{B}_{i} ; \quad i, j=1,2 ; \quad \gamma_{1}=\beta_{1}, \quad \gamma_{2}=\alpha^{\prime}{ }_{1}
$$

where

$$
{ }_{1} \mathcal{M}=\left[\begin{array}{cc}
2 \Delta_{p} & 2 \\
0 & 2 \Delta_{p}
\end{array}\right] \quad{ }_{1} \mathcal{B}=\left[\begin{array}{c}
1 \\
(\hat{A}-1)
\end{array}\right]
$$

which results in:

$$
\left[\begin{array}{c}
\beta_{1} \\
\alpha^{\prime}{ }_{1}
\end{array}\right]=\frac{1}{2 \Delta_{p}^{2}}\left[\begin{array}{c}
\Delta_{m}-\Delta_{n} \\
\Delta_{p}(\hat{A}-1)
\end{array}\right]
$$

It is not surprising that $\alpha^{\prime}{ }_{1}=\alpha_{1}$.

For the second level we have

$$
\left|2, \Delta_{p}^{\prime}>=\left(\alpha_{2}^{\prime} L_{-1}^{2}+\alpha_{3}^{\prime} L_{-2}\right)\right| \Delta_{p}^{\prime}>+\left(\beta_{2} L_{-1}^{2}+\beta_{3} L_{-2}\right) \mid \Delta_{p}>
$$

Using (82 85), we see that $\alpha_{2}^{\prime}, \alpha_{3}^{\prime}, \beta_{2}$ and $\beta_{3}$ satisfy the following system of equations:

$$
{ }_{2} \mathcal{M}_{i j} \gamma_{j}={ }_{2} \mathcal{B}_{i} ; \quad i, j=1,2,3,4 ; \quad \gamma_{1}=\beta_{2}, \quad \gamma_{2}=\beta_{3}, \gamma_{3}=\alpha_{2}^{\prime}, \gamma_{4}=\alpha^{\prime}{ }_{3}
$$

where

$$
{ }_{2} \mathcal{M}=\left[\begin{array}{cccc}
4 \Delta_{p}+2 & 3 & 4 & 0 \\
6 \Delta_{p} & 4 \Delta_{p}+\frac{c}{2} & 6 & 4 \\
0 & 0 & 4 \Delta_{p}+2 & 3 \\
0 & 0 & 6 \Delta_{p} & 4 \Delta_{p}+\frac{c}{2}
\end{array}\right] \quad{ }_{2}^{\mathcal{B}}=\left[\begin{array}{c}
\hat{A} \beta_{1}+\alpha_{1} \\
1 \\
\hat{A} \alpha_{1} \\
\hat{B}
\end{array}\right]
$$


These result in:

$$
\left[\begin{array}{c}
\beta_{2} \\
\beta_{3} \\
\alpha^{\prime}{ }_{2} \\
\alpha^{\prime}{ }_{3}
\end{array}\right]=\frac{1}{\Delta_{1}^{2}}\left[\begin{array}{c}
\hat{C}\left[\hat{A}\left(\Delta_{1} \beta_{1}-4 \hat{C} \alpha_{1}\right)+\Delta_{1} \alpha_{1}-24 \hat{B}\right]+3\left[c\left(3 \hat{A} \alpha_{1}+2 \hat{B}\right)+10 \hat{B}-\Delta_{1}\right] \\
\Delta_{1}\left(\hat{D}-6 \Delta_{p}\left(\hat{A} \beta_{1}+\alpha_{1}\right)\right)+6 \hat{A} \alpha_{1}\left(16 \Delta_{p}^{2}-c\right)-4 \hat{B}(\hat{D}-9) \\
\hat{A} \alpha_{1} \Delta_{1} \hat{C}-3 \hat{B} \Delta_{1} \\
-6 \hat{A} \alpha_{1} \Delta_{1} \Delta_{p}+\hat{B} \Delta_{1}\left(4 \Delta_{p}+2\right)
\end{array}\right]
$$

For the third level, using eqs. (82-85), we have:

$$
\left|3, \Delta_{p}>=\left(\beta_{4} L_{-1}^{3}+\beta_{5} L_{-1} L_{-2}+\beta_{6} L_{-3}\right)\right| \Delta_{p}>+\left(\alpha_{4}^{\prime} L_{-1}^{3}+\alpha_{5}^{\prime} L_{-1} L_{-2}+\alpha_{6}^{\prime} L_{-3}\right) \mid \Delta_{p}^{\prime}>
$$

where $\alpha_{i}^{\prime}$ and $\beta_{i}(i=4,5,6)$ satisfy the following system of equations:

$$
{ }_{3} \mathcal{M}_{i j} \gamma_{j}={ }_{3} \mathcal{B}_{j}, \quad i, j=1, \cdots, 6
$$

$\gamma_{i}^{\prime}$ 's $(i=1,2,3)$ denote $\beta_{i}^{\prime}$ 's $(i=4,5,6)$ and $\gamma_{i}^{\prime}$ 's $(i=4,5,6)$ denote $\alpha_{i}^{\prime \prime}$ s $(i=4,5,6)$, respectively. The exact expression for ${ }_{3} \mathcal{M}_{i}$ and ${ }_{3} \mathcal{B}_{i}$ are:

$$
\begin{aligned}
& { }_{3} \mathcal{M}=\left[\begin{array}{cccccc}
18 \Delta_{p}+6 & 4 \Delta_{p}+9+\frac{c}{2} & 5 & 18 & 4 & 0 \\
24 \Delta_{p} & 16 \Delta_{p}+2 c & 6 \Delta_{p}+2 c & 24 & 16 & 6 \\
6 \Delta_{p}+6 & 2 \Delta_{p}+7 & 4 & 6 & 2 & 0 \\
0 & 0 & 0 & 18 \Delta_{p}+6 & 4 \Delta_{p}+9+\frac{c}{2} & 5 \\
0 & 0 & 0 & 24 \Delta_{p} & 16 \Delta_{p}+2 c & 6 \Delta_{p}+2 c \\
0 & 0 & 0 & 6 \Delta_{p}+6 & 2 \Delta_{p}+7 & 4
\end{array}\right] \\
& { }_{3} \mathcal{B}=\left[\begin{array}{c}
(\hat{B}+1) \beta_{1}+\alpha_{1} \\
1 \\
(\hat{A}+1)\left(\beta_{2}+\beta_{3}\right)+\left(\alpha_{2}+\alpha_{3}\right) \\
(\hat{B}+1) \alpha_{1}^{\prime} \\
\left(\hat{B}+\Delta_{n}\right) \\
(\hat{A}+1)\left(\alpha_{2}^{\prime}+\alpha_{3}^{\prime}\right)
\end{array}\right]
\end{aligned}
$$

In the above results, three significant remarks are inorder:

1- In the diagonal blocks of ${ }_{i} \mathcal{M}$, there are two copies of relevant ordinary ${ }_{i} \mathcal{N}$. 
2- The upper off diagonal block of ${ }_{i} \mathcal{M}$ is the derivative of the diagonal block with respect to $\Delta_{p}$ and the lower off-diagonal block is zero.

This is another evidence insupport of the fact that logaritmic operator together with ordinary operator form the basis of Jordan cell for $L_{0}$.

Hence,

$$
{ }_{i} \mathcal{M}=\left[\begin{array}{cc}
{ }_{i} \mathcal{N} & \partial_{\Delta_{p}}{ }_{i} \mathcal{N} \\
0 & { }_{i} \mathcal{N}
\end{array}\right] \quad{ }_{i} \mathcal{B}=\left[\begin{array}{l}
{ }_{i} \mathcal{D} \\
{ }_{i} \mathcal{A}
\end{array}\right]
$$

where $\partial_{\Delta_{p}}=\frac{\partial}{\partial_{\Delta_{p}}},{ }_{i} \mathcal{A}$ is the same as in the last subsection and ${ }_{i} \mathcal{D}$ is a column matrix with elements which resemble the first half of ${ }_{i} \mathcal{B}$ in eqs. (88,92).

Finally, $\beta_{i}$ s and $\alpha_{i}^{\prime}$ s are as follows:

$$
\begin{gathered}
{\left[\alpha_{i}^{\prime}\right]=\left[\alpha_{i}\right]={ }_{i} \mathcal{N}^{-1}{ }_{i} \mathcal{A}} \\
{\left[\beta_{i}\right]={ }_{i} \mathcal{N}^{-1}{ }_{i} \mathcal{D}+\left(\partial_{\Delta_{p}}{ }_{i} \mathcal{N}^{-1}\right){ }_{i} \mathcal{A} .}
\end{gathered}
$$

3- Most significant remark is that in the expressions $(89,92,97)$, first half of elements is partial derivative of second half of elements with respect to $\Delta_{p}$. This means that ${ }_{i} \mathcal{D}=$ $\partial_{\Delta_{p}}\left({ }_{i} \mathcal{A}\right)$.

Finaly we can conclude as follows:

$$
\left|n, \Delta^{\prime}>=\alpha_{k_{1}, k_{2}, \ldots} L_{-1}^{k_{1}} L_{-2}^{k_{2}} L_{-3}^{k_{3}} \cdots\right| \Delta_{p}>+\left(\partial_{\Delta_{p}} \alpha_{k_{1}, k_{2}, k_{3} \ldots} L_{-1}^{k_{1}} L_{-2}^{k_{2}} L_{-3}^{k_{3}} \cdots \mid \Delta_{p}^{\prime}>\right.
$$

where $\sum m k_{m}=n$. It seems that appearance of $\partial_{\Delta_{p}}$ has more important rule in logarithmic operator.

\section{Acknowledgment:}

We would like to acknowledge A. Aghamohammadi, M. Khorrami, M.R. Mohayaee, V. Karimipour and S. Rouhani for their useful discussions. The authors are indebted to A. Morozov for his continuous encouragement. 


\section{References}

[1] V. Gurarie, Nucl. Phys. B410[FS] 535 (1993).

[2] H. Saleur, Yale Preprint YCTP-P38-91, 1991.

[3] L. Rozansky and H. Saleur, Nucl. Phys. B376 461 (1992).

[4] G. Cardy, UCSB Preprint UCSBTH-91-56, 1991.

[5] X.G. Wen, Y.S. Wu and Y. Hatsugai, Nucl. Phys. B422[FS] 476 (1994).

[6] M.R. Rahimi Tabar and S. Rouhani, Annals of Phys. 246446 (1996).

[7] M.R. Rahimi Tabar and S. Rouhani," The Alf"ven Effect and Conformal Field Theory" IPM Preprint IPM-094-95 hep-th/9507166] to appear in Nuovo. Cimento B.

[8] M.R. Rahimi Tabar and S. Rouhani, Europhys. Lett. 37 (1996) 447.

[9] M. A. I. Flohr, Nucl. Phys. B482 (1996) 567.

[10] M.R. Rahimi Tabar and S. Rouhani, Phys. Lett. A224 (1997) 331.

[11] M. R. Gaberdiel, H.G. Kausch, Nucl. Phys. B477 (1996) 293.

[12] A. Bilal and I.I. Kogan, Princton Preprint PUPT-1482 [hep-th/9407151]; Nucl. Phys. B449 569 (1995).

[13] Ch. Schmidhuber, Nucl. Phys.404 342 (1993); Nucl. Phys. B453 156 (1995).

[14] I.I. Kogan and N.E. Mavromatos, Oxford Preprint-95-50 P , hep-th/9512210.

[15] J.S. Caux, I.I. Kogan and A.M. Tsvelik, Preprint OUTP-95-62 S, hep-th/9511134.

[16] Z. Maassarani, D. Serban," Non-Unitary Conformal Field Theory and Logarithmic Operators for Disordered Systems", preprint SPHT-T96/037 (1996), hep-th/9605062.

[17] R. Blumenhagen, W. Eholzer, A. Honecker, K. Hornfeck, R. Hubel, Phys. Lett. B332 51 (1994), Int. Jour. Mod. Phy. A10 2367 (1995).

[18] J. Ellis, N.E. Mavromatos and D.V. Nanopoulos," D Branes from Liouville Strings" CERN-TH/96-81, hep-th/9605046.

[19] M.A.I. Flohr, Int. J. Mod. Phys. A11 (1996) 4147. 
[20] M.A.I. Flohr " On Fusion Rules in Logarithmic Conformal Field Theories" IASSNSHEP-96/54, hep-th/9605151.

[21] A.M. Belavin, A.M. Polyakov and A.B. Zamolodchikov, Nucl. Phys. B241 333 (1984).

[22] H. Bateman, "Higher Transcendental Functions", Vol. I, (McGraw-Hill, New York, 1953)pp. 68-69.

[23] A. Shafiekhani Mod. Phy. Lett. A9 3273 (1994).

[24] C.N. Pope, L.J. Romans and X. Shen, Phys. Lett. B236 173 (1990).

[25] C.N. Pope, X. Shen and L.J. Romans, Nucl. Phys. B339 191 (1990).

[26] W.S. Chung and A. Shafiekhani, Phys. Lett. B381 68 (1996).

[27] M.R. Rahimi Tabar, A. Aghamohammadi and M. Khorrami, "The logarithmic conformal field theory" to appear in Nucl. Phys. B 